\title{
NUEVAS INSCRIPCIONES LATINAS DE LA NECRÓPOLIS GADITANA
}

\author{
Antonio Ruiz Castellanos \\ Marcos A. Martelo Fernández \\ Universidad de Cádiz
}

Recibido: 4/03/2015

Revisado: 6/04/2015

RESUMEN

En este trabajo se estudian nuevas inscripciones de la necrópolis romana de Cádiz, procedentes de las excavaciones en las calles María Soledad y General Ricardos.
Aceptado: 6/04/2015

Publicado: 30/05/2015

\section{Abstract}

In this work are studied new latin inscriptions of the Roman necropolis of Cadiz, from the excavations in the streets María Soledad and General Ricardos.
Palabras Clave

Inscripciones Latinas; Necrópolis; Cádiz.
KEYWORDS

Latin Inscriptions; Necropolis; Cádiz.

antonio.ruizcastellanos@uca.es

marcos.martelo@uca.es 
Presentamos en este trabajo dos grupos de inscripciones halladas en la zona de la necrópolis gaditana ${ }^{1}$. Los primeros 10 fragmentos fueron hallados en el año 2011 en las excavaciones preceptivas del solar próximo a la torre de Telefónica en la c/ María Soledad n ${ }^{0} 4$ de Cádiz, y la segunda es una inscripción funeraria dedicada a Bruttia, encontrada hace años en la misma zona (c/ Gral. Ricardos), que procede de una colección particular de Cádiz (figura 1). la constatación de dos fases de necrópolis, una tardo-púnica del s. II a.C. (Niveau de Villedary y Martelo Fernández, 2014, 160) y una romana altoimperial del s. I d.C.

La fase romana alto-imperial se iniciaría en el s. I d.C. localizándose una serie de estructuras hidráulicas, canalizaciones, pileta y pozo, y dos inhumaciones en fosa simple. En momentos avanzados del s. I d.C., se situarían las 12 fosas romanas documentadas, que se sumarían a las 3

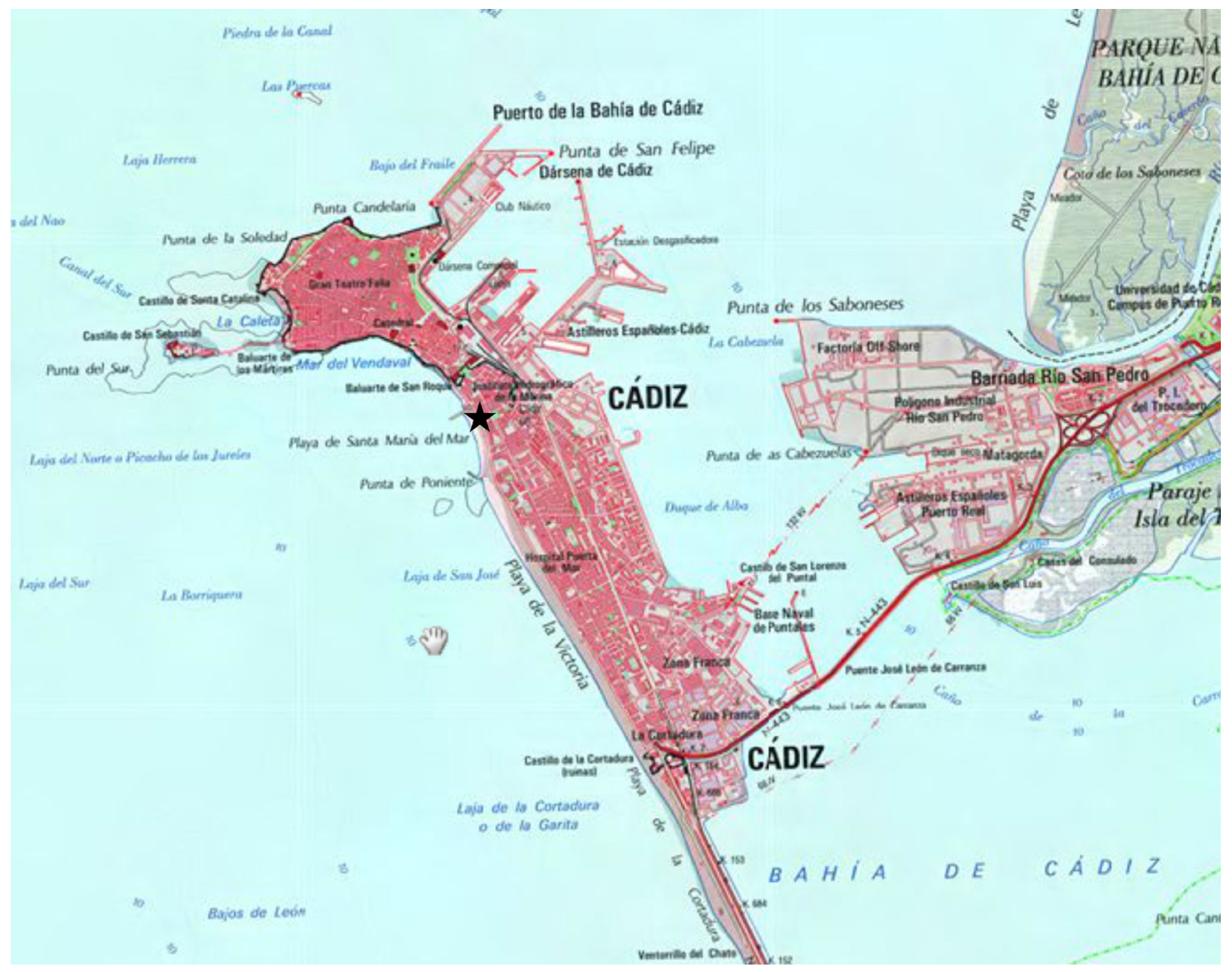

Fragmentos Hallados EN LA EXCAVACión DE LA C/ MARÍA SOLEDAD N ${ }^{0} 4$

La excavación arqueológica realizada en 2011 en el solar de la calle Santa María Soledad $\mathrm{n}^{\mathrm{o}} 4$, en la zona extramuros de Cádiz, ha dado como resultado

1 Este trabajo es resultado del proyecto FFI201239395-C02-01concedido por el Ministerio de Economía y Competitividad. Agradecemos a Helena Gimeno su supervisión. localizadas en el control perimetral previo. Estas fosas presentan abundantes materiales cerámicos y óseos y, en ocasiones, cortan estructuras hidráulicas anteriores. Entre las cerámicas, junto a fragmentos de cerámica común y de ánforas, sobre todo de Dressel 7/11, sobresalen copas y cuencos de Terra Sigillata Itálica y Sudgálica, vasos Paredes Finas y vasos con forma de ave (askoi). Las características formales de estas estructuras negativas, su cota 
y posición estratigráfica, que corta niveles más antiguos, así como los elementos que las rellenan tales como huesos de fauna, los askoi, los opérculos o tapaderas, clavos de hierro y bronce y restos de ungüentarios de vidrio, nos llevan a identificarlas como vertederos relacionados con la necrópolis. En alguna de estas fosas (UE15, UE100) aparecen algunos fragmentos de inscripciones en mármol (Inscripciones 6 y 7). También se encuentra algún fragmento de inscripción (Inscripciones 9 y 10) en el relleno de un pozo (UE36) amortizado en estos momentos del s. I d.C.

Cortandoalguna deestas fosas y alguna estructura hidráulica anterior se documentan una serie de cimentaciones, realizadas principalmente con cantos, que corresponderían a una fase constructiva posterior arrasada, de la que no tenemos apenas más vestigios, y que aparece cubierta por un nivel de abandono (UE3), donde entre otros materiales alto-imperiales y bajo-imperiales, se hallaron varios fragmentos de inscripciones (Inscripciones 1, 2, 3,4, 5 y 8$)$.

Las inscripciones se conservan en el Museo de Cádiz donde las vimos y fotografiamos en el año 2011.

1. Placa de mármol rota en todos sus lados menos por abajo donde conserva borde original, con la parte posterior lisa. Mide (7) x (3,5) x 1,8 cm (figura $2,1)$. Las letras, de buena factura, son capitales cuadradas con remates muy marcados de una altura de $5 \mathrm{~cm}$. Corresponde a la unidad estratigráfica SMS4/11/AA/C4/UE3/EPI-1 (Museo de Cádiz, inv. n. Depósito: DJ-13-2).

- . - . -

[- - ? h(ic)] s(it-) • e(st) [s(it)t(ibi) t(erra)l(evis)]

2. Placa de mármol quebrada por todos los lados, con la parte posterior lisa. Mide $(3,7)$ x (5) x 2,2 $\mathrm{cm}$ (figura 2,2). Las letras, de muy buena factura, son capitales cuadradas de una altura de $2,7 \mathrm{~cm}$. Corresponde a la unidad estratigráfica SMS4/11/ AA/C4/UE3/EPI-2 (Museo de Cádiz, inv. n. Depósito: DJ-13-2).

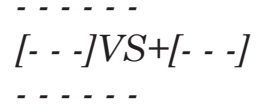

La cruz puede que sea la línea recta transversa superior de una $\mathrm{T}$.

3. Placa de mármol rota por todos los lados, con la parte posterior lisa. Mide (7) x (8) x $5 \mathrm{~cm}$. Las letras son capitales cuadradas de una altura de 2,3 $\mathrm{cm}$ (figura 2,3). Interpunción circular. Corresponde a la unidad estratigráfica SMS4/11/AA/C4/UE3/ EPI-3 (Museo de Cádiz inv. n. Depósito: DJ-13-2).

$$
\begin{aligned}
& \text { an(norum) }[---] \\
& h(\text { ic }) \cdot s(i t-)[e(s t) s(\text { it }) t(\text { ibi }) t(\text { erra }) l(\text { evis })]
\end{aligned}
$$

4. Placa de mármol rota por todos los lados, con la parte posterior lisa de (6) x (3) x 1,8 cm. La altura de las letras es de $2,5 \mathrm{~cm}$ (figura 2,4). La interpunción es en forma de hedera. Corresponde a la unidad estratigráfica SMS4/11/AA/C4/UE3/ EPI-4 (Museo de Cádiz, inv. n. Depósito: DJ-13-2).

$$
\text { - } h(\text { ic) }[s(i t-) e(s t) s(i t) t(\text { ibi) } t(\text { erra) } l(\text { evis) }]
$$

5. Placa de mármol quebrada por arriba y por los lados derecho e izquierdo, con la parte posterior lisa. Mide $(4,8) \times(3,8) \times 2,5 \mathrm{~cm}$ (figura 2,5). La altura de las letras es de 2,9 y la interpunción es en forma de hedera. Corresponde a la unidad estratigráfica SMS4/11/AA/C4/UE3/EPI-5 (Museo de Cádiz. inv. n. Depósito: DJ-13-2).

$$
\text { [- - - - }
$$

La cruz es un trazo oblicuo descendente, posiblemente una A o M.

6. Placa de mármol con vetas azules recortada por arriba, a la derecha, y, por abajo, en forma de ángulo con la parte posterior sin alisar. Mide (11) x (10) x 2-1,5 cm (figura 5, 1). Las letras capitales cuadradas tienen una altura aproximada de $5 \mathrm{~cm}$. Corresponde a la unidad estratigráfica SMS4C/11/ SSO/UE15/EPI (Museo de Cádiz inv. n. Depósito: DJ-13-2).

$$
\text { Ag[- - ] }
$$


Por el tipo de letra se fecha entre época flavia y trajanea.

7. Placa de mármol rota a la derecha y por debajo con la parte posterior lisa. Mide $(7) \mathrm{x}(7,2)$ $x 1,6 \mathrm{~cm}$ (figura 3). Las letras tienen una altura de $2 \mathrm{~cm}$, en la primera línea y de 4,2 en la segunda donde conservan restos de minio y la interpunción es en forma de hedera. Corresponde a la unidad estratigráfica SMS4/11/AA/C4/UE100/EPI-6 (Museo de Cádiz, inv. n. Depósito: DJ-13-2).

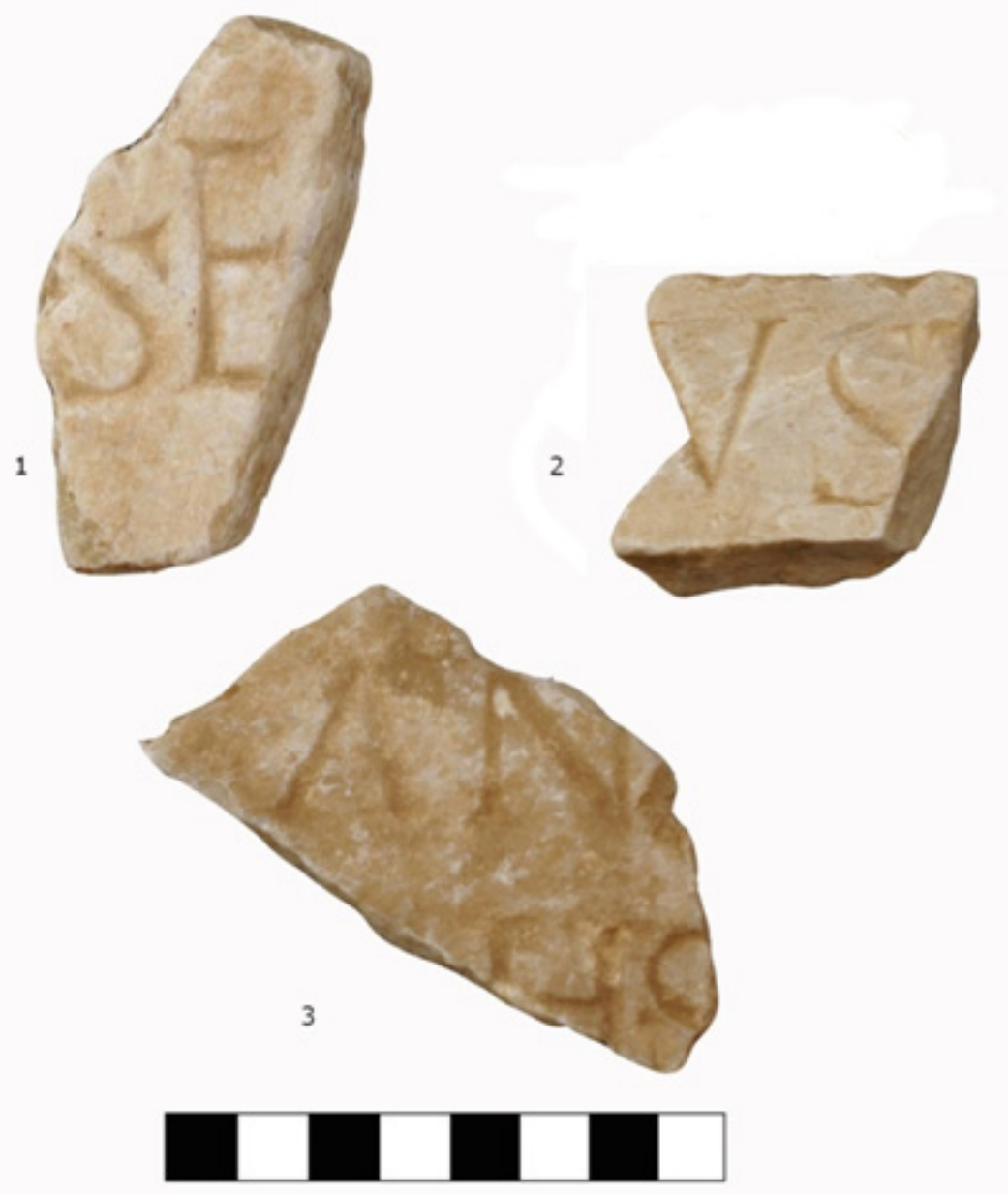

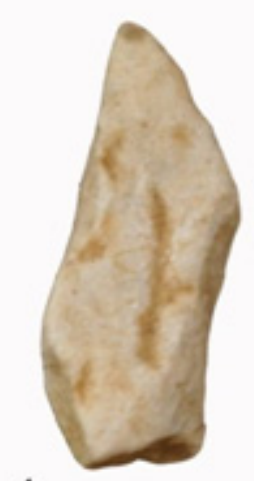

4

$$
\begin{aligned}
& \text { M[emoria? - - -] } \\
& L(u c i) ・ S[---]
\end{aligned}
$$




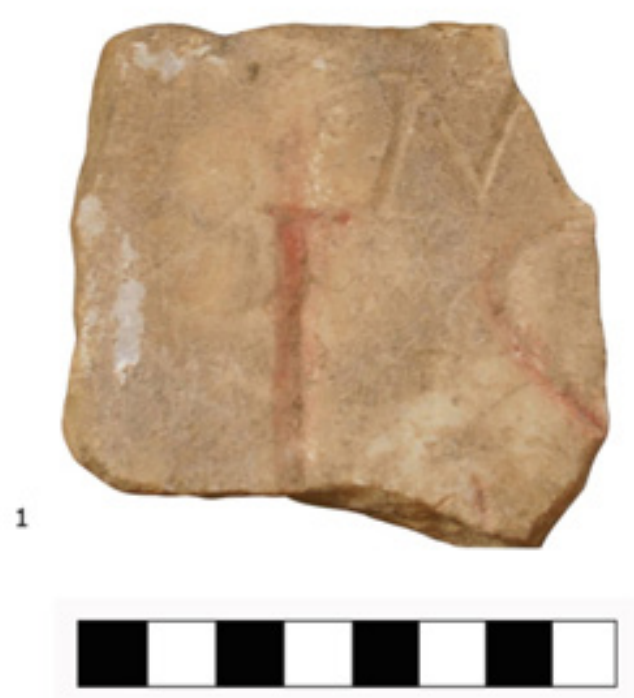

Figura 3. SMS4/11/AA/C4/UE100: EPI-6

Debido a que la $\mathrm{M}$ es comienzo de línea y a que en Cádiz dos epígrafes comienzan por el término Memoria (González Fernández, 1982, 125 y 163) preferimos esta restitución a un posible $M$ (anibus) $D$ (is) aunque no podemos rechazarlo ya que hay testimonios de esta inversión. Por ejemplo en Béjar (Salamanca; CIL II, 882), en Arana (Alava, HEp-3, 14 M(anibus), sólo en la civitas Igaeditanorum (AE 2002, 677).

8. Placa de mármol quebrada por todos los lados, con la parte posterior lisa. Mide $(6,2)$ x $(3,5)$ x 1,5 $\mathrm{cm}$ (figura 5,2). Las letras capitales cuadradas tienen una altura de $2,8 \mathrm{~cm}$ en la primera línea y 2,5 en la segunda. Corresponde a la unidad estratigráfica SMS4C/11/SNE/UE3B/EPI (Museo de Cádiz, inv. n. Depósito: DJ-13-2).

$$
\begin{gathered}
2--- \\
{[---]+[--]} \\
{[--] I I[---]}
\end{gathered}
$$

En la primera línea la primera cruz es una I o una $\mathrm{T}$, la segunda parece más bien una $\mathrm{O}$ que una $\mathrm{Q}$. Puede que pertenezcan al final de un nomen -io o al comienzo de un cognomen To-. En la segunda línea los dos signos podrían formar parte del numeral de la edad.
9. Placa de mármol recortada por todos sus lados menos por abajo donde conserva borde original. La parte posterior ha sido alisada con un escalpro dentado. Mide $(7,5)$ x $(10,1)$ x 1,5 cm (figura 4,1). Las letras capitales cuadradas tienen una altura entre 4 y 3,8. Corresponde a la unidad estratigráfica SMS4/11/POZO/UE36/EPI-7 (Museo de Cádiz, inv. n. Depósito: DJ-13-2).

$$
[---] \mathrm{Vic}[---]
$$

Por la posición que ocupa quizá podría ser inicio de un cognomen que empiece por Vict-. Por el tipo de letra segunda mitad del s. I o siglo II.

10. Placa de mármol recortada por todos los lados. En la parte posterior se observan foramina ciegos, distribuidos de forma alternante y regular, quizás con la intención de fijar la placa con algún cemento. Mide $(6,5)$ x (9) x $4 \mathrm{~cm}$ (figura 4,2). Las letras tienen una altura de $5 \mathrm{~cm}$ aproximadamente. Corresponde a la unidad estratigráfica SMS4/11/ POZO/UE36/EPI-8 (Museo de Cádiz, inv. n. Depósito: DI-13-2).
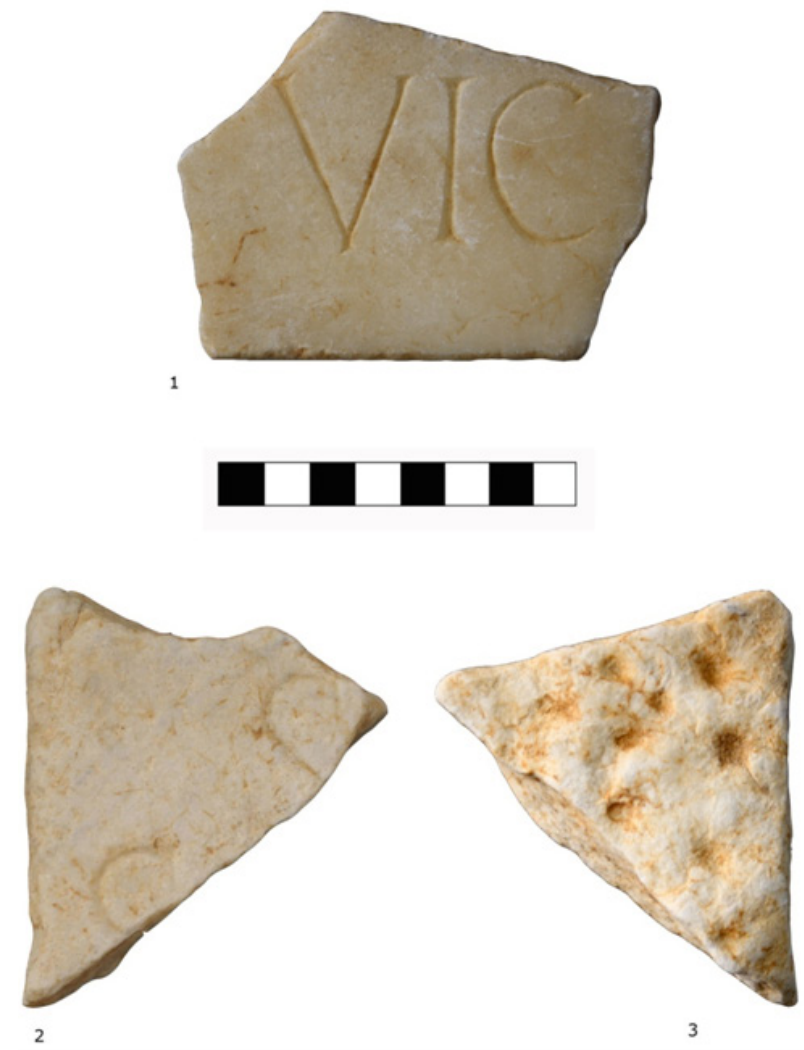

Figura 4. SMS4/11/POZO/UE36: 1, EPI-7; 2 y 3: EPI-8 


$$
\begin{aligned}
& {[---]+[---]} \\
& {[--] S(--) \cdot[--]}
\end{aligned}
$$

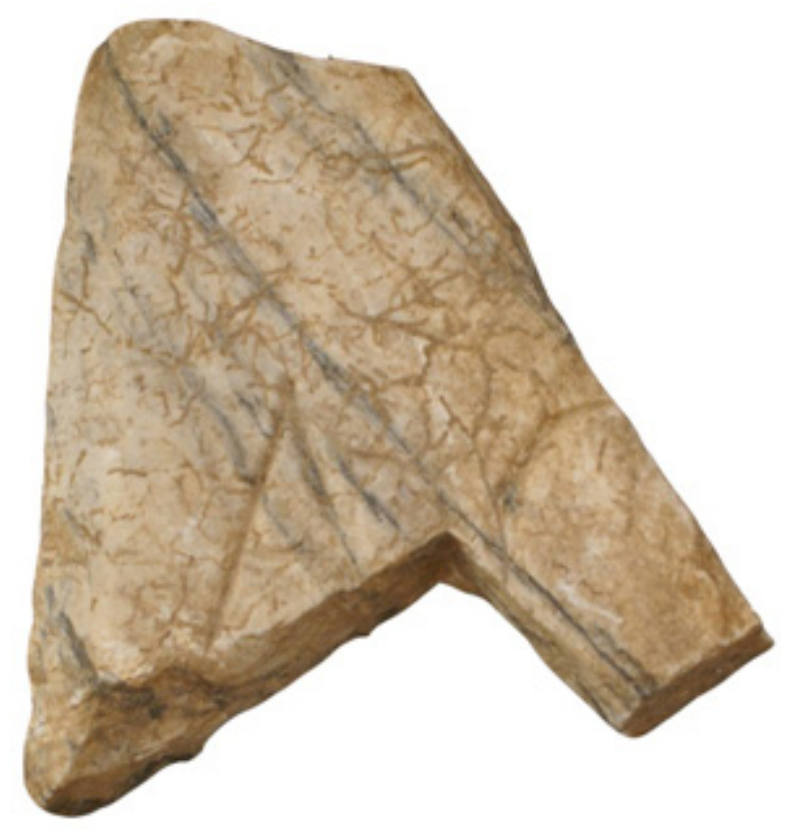

1

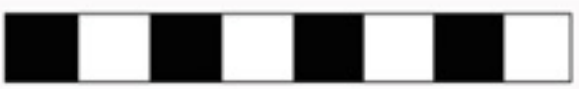

2

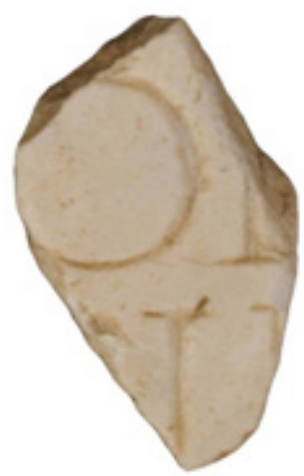

Figura 5. 1: SMS4C/11/SSO/UE15/EPI 9; y 2: SMS4C/11/ SNE/UE3B/EPI 10
INSCRIPCIÓN DE C/ GENERAL RICARDOS.

Es una pequeña placa de mármol blanco de aspecto irregular debido a que el contorno está roto en el lado izquierdo y bastante mellado, especialmente en los ángulos superior e inferior derechos. Por la parte posterior la superficie es lisa. Sus medidas son $(16,3)$ x (18) y $3 / 2 \mathrm{~cm}$. Debajo de la última línea se observa un resalte que si bien podría corresponder a la superficie que enmarcaba un campo epigráfico rebajado, tampoco es imposible que corresponda simplemente al estado del soporte previo a su utilización para la inscripción. En efecto, otras características de la placa nos hacen sospechar que para Bruttia se reutilizó quizá un recorte sobrante del taller de un marmolista. Así, entre los dos primeros renglones y atravesando parcialmente las tres primeras letras del segundo, se aprecian los restos de una línea que si bien parece a primera vista de pautado, curiosamente, no funciona como tal en el texto y debió haber sido trazada con otra intención. La impaginación, bastante rudimentaria, se realizó respecto a un eje central, excepto para la línea 5. Las letras de surco poco profundo, irregulares, de calidad mediocre y ejecución imprecisa parecen realizadas a mano alzada (nótese, por ejemplo, que en la 1. 4, la palabra vix(it) presenta ondulaciones en los trazos oblicuos de N, V y X). Su altura es de 1,2 cm en las líneas 1 y 5 y de $2 \mathrm{~cm}$ en las líneas 2-4. Los espacios interlineales son de $1,5 \mathrm{~cm}$ y no hay signos de interpunción. El estado de conservación es bueno.

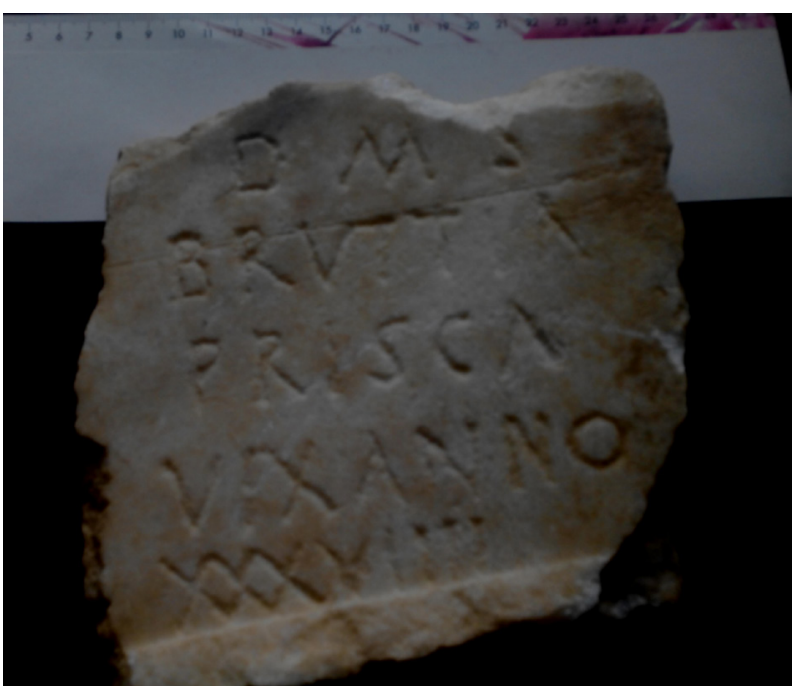

Figura 6. Inscripción de la c/ General Ricardos.

El texto dice 
$D$ (is) $M$ (anibus) s(acrum)

Bruttia

Prisca

vix(it) anno(s)

XXXVIIII

Hay que observar en la línea 4 la caída de la $S$ final en la palabra ANNO, un fenómeno habitual bien documentado (Carnoy, 1971: 179-199; Bassols de Climent, 1973: 192-193, $\mathrm{n}^{\mathrm{o}}$ 256-257). El gentilicio Bruttia, bien conocido por ser de una destacada familia senatorial con conexiones con los Antoninos (Mennen, 2011, 90-91), no es muy común en Hispania. Lo encontramos en la Baetica en Celti (HEp 10, 533 y 534), en la Hispania Citerior en Segobriga (CIL II $3121=$ Abascal y Cebrían , 2002, 00081) y Tarraco (CIL II $2 / 14-3$, 1490), y, en Lusitania en Mirtilis (CIL II, 5178) y en la Civitas Igaeditanorum (HEp 13, 883).

Por la onomástica y el formulario se podría fechar a finales del siglo II o en el III.

BiBLIOGRAFÍA

Bassols de Climent, M. (1973), Fonética Latina, Madrid.

Carnoy, A.J. (1971), Le latin d'Espagne d'après les inscriptions, G. Olms, Hildesheim, 179-199

González Fernández, J. (1982): Inscripciones romanas de la provincia de Cádiz, Cádiz, Excma. Diputación provincial de Cádiz.

Abascal, J. M. y Cebrián, R. (2002), "Inscripciones romanas de Segobriga (1999-2001 e inéditas)”, Saguntum, 34, 151-186

Mennen, I. (2011), Power and status in the Roman Empire, AD 193-284, Leiden.

Niveau de Villedary, A.M. y Martelo Fernández, M. A. (2014), "Puntualizaciones sobre los "pebeteros en forma de cabeza femenina" tardopúnicos. A propósito de un hallazgo reciente", Imagen y culto en la Iberia prerromana II. Nuevas lecturas sobre los pebeteros en forma de cabeza femenina, Spal Monografías, 18, 155173. 
